Scientific Journal of Hamadan Nursing \& Midwifery Faculty - ISSN 2008-2800

\title{
Effects of Matricaria Chamomilla on the Severity of Nausea and Vomit- ing Due to Chemotherapy
}

\author{
Fatemeh Borhan $^{1}$, Ali Naji ${ }^{*}$, Mehdi Molavi Vardanjani ${ }^{3}$, Lida Sasani ${ }^{4,5}$
}

1. MSc Student, Faculty of Nursing and Midwifery,, Islamic Azad University of Isfahan, Khorasgan Branch, Isfahan, Iran

2. Associate Professor, Faculty of Nursing and Midwifery, Islamic Azad University of Isfahan, Khorasgan Branch, Isfahan, Iran

3. Instructor, Faculty of Nursing and Midwifery, Hamadan University of Medical Sciences, Hamadan, Iran

4. Instructor, Member of Young and Elite Researchers Club, Islamic Azad University of Isfahan, Khorasgan Branch, Isfahan, Iran

5. Instructor, Faculty of Nursing and Midwifery, Islamic Azad University of Isfahan, Khorasgan Branch, Isfahan, Iran

\begin{tabular}{lll}
\hline Article Info & Abstract \\
\hline
\end{tabular}

Received: $\quad 2017 / 03 / 5$

Accepted: $\quad$ 2017/07/3

Published Online 2017/07/17

DOI:

Original Article

Use your device to scan and read the article online

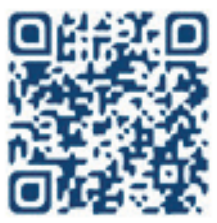

Introduction: Uncontrollable nausea and vomiting can cause delay in chemotherapy and affects patient's life. Since Matricaria chamomilla seems to be effective in reducing nausea and vomiting, this study aimed to determine the effect of its extract on the severity of nausea and vomiting due to chemotherapy.

Methods: This quasi experimental study was conducted on the patients receiving chemotherapy in Chemotherapy Wards of Zahedan Tamin Ejtemaee Hospitals in 2015. In doing so, 60 patients were selected through convenience-sampling method and assigned into intervention and control groups. Two hours before chemotherapy, the intervention group was given Matricaria chamomilla extract while the control patients were given distilled water as placebo. Nausea rate was measured using Visual Analogue Scale (VAS) 0, 2, 6, and 12 hours after chemotherapy.

Results: In the beginning, there was no significant difference between the mean scores of nausea of two groups $(P=0.4)$; however, 2 hours after the chemotherapy, nausea score increased in both groups and it was significantly higher in the control group. $(P=0.034)$. After 6 and 12 hours, the mean scores of nausea in both groups declined; however, the control patients' mean score was constantly and significantly higher. There was no significant difference between the two groups concerning vomiting $(P>0.05)$.

Conclusion: The results indicated that consuming Matricaria chamomilla extract can reduce nausea due to chemotherapy while it has no effect on vomiting.

Keywords: Chemotherapy; Matricaria Chamomilla; Nausea; Vomiting

Copyright $($ C 2017, Sci J Hamadan Nurs Midwifery Fac. This is an open-access article distributed under the terms of the Creative Commons Attribution-noncommercial 4.0 International License which permits copy and redistribute the material just in noncommercial usages, provided the original work is properly cited.

How to Cite This Article:

Borhan F, Naji A, MolaviVardanjani M, Sasani L. Effects of Matricaria Chamomilla on the Severity of Nausea and Vomiting Due to Chemotherapy. Sci J Hamadan Nurs Midwifery Fac. 2017; 25 (4): 140-146 


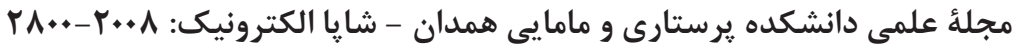

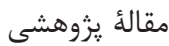

\section{بررسى تأثير عصارة گياه بابونه بر شدت تهوع و استفراغ وابسته به شيمىدرمانى}

\section{فاطمه برهان'، على ناجى ז"، مهلدى مولوى وردنجانى"، ليدا ساسانى}

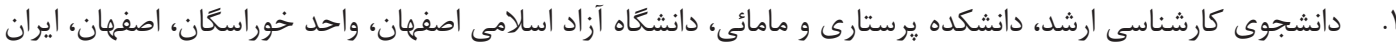

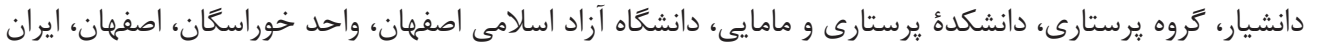

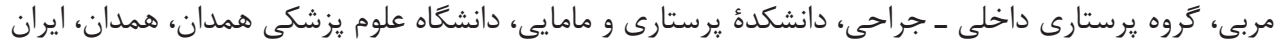

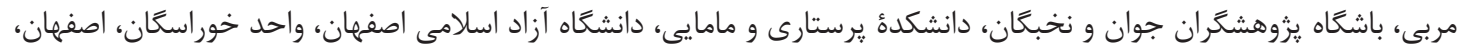

مربى، كروه يرستارى، دانشكدة يرستارى و مامايى، دانشگاه آزاد اسلامى اصفهان، واحد خوراسكان، اصفهان، ايران

جانبى مثل ريزش موهاى بدن، دردهاى عضلانى، اختلالات

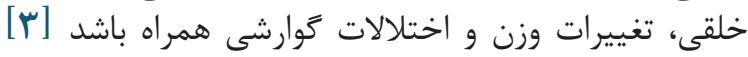

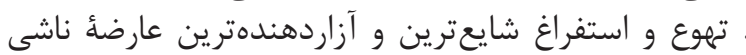

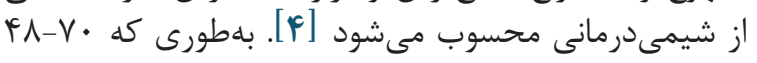

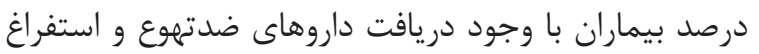

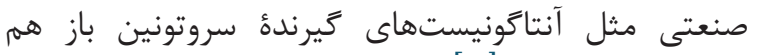

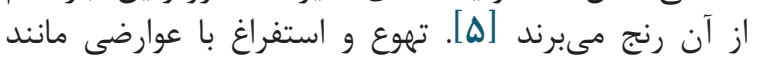

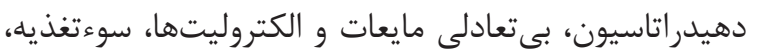

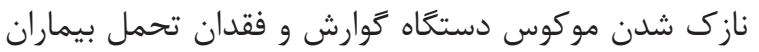

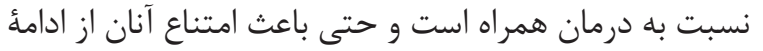

1. Visual Analog Scale
سرطان يك بيمارى در حال گسترش است. براساس

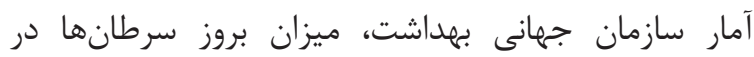

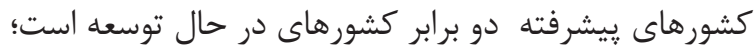

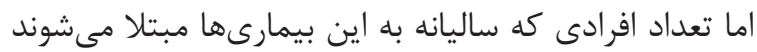

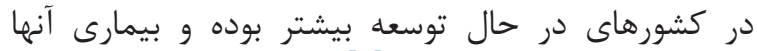

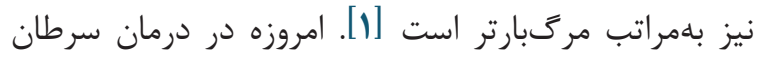

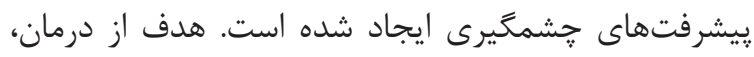

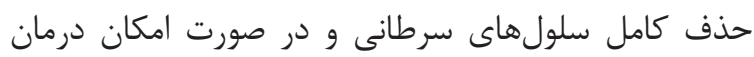
كنترلى يا تسكينى سرطان براى كاهش نس نشانه

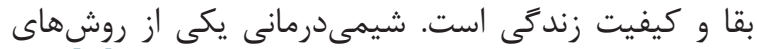

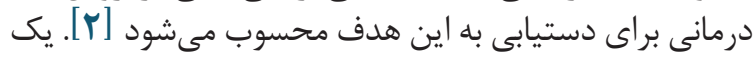

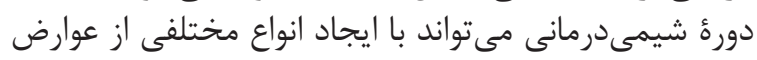




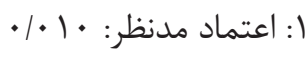

P

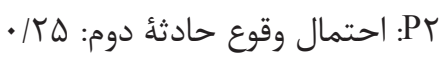

r : حداكثر خطاى قابلقبول: p-p

ابتلا به يكى از انواع سرطانها، استفاده از شيمىدرمانى

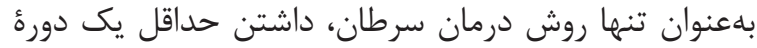

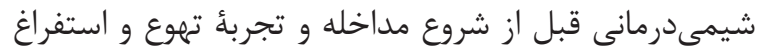

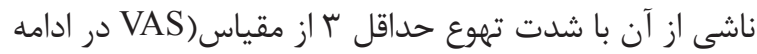

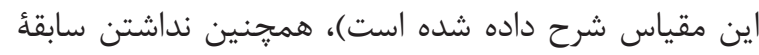

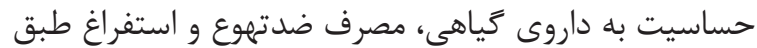

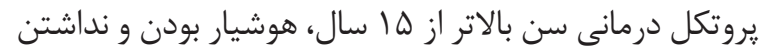

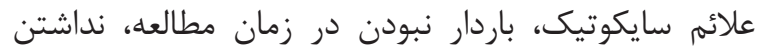

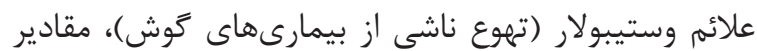

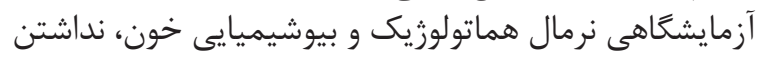

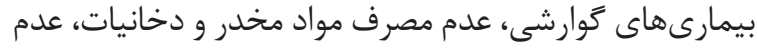

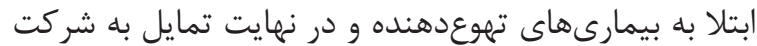

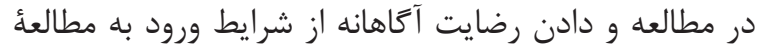

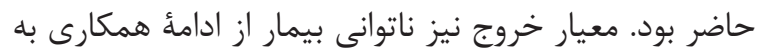

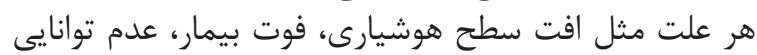

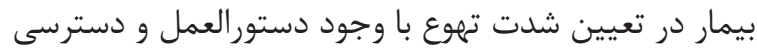

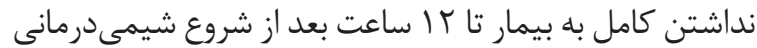

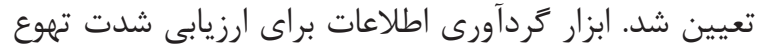

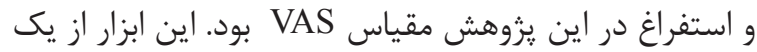

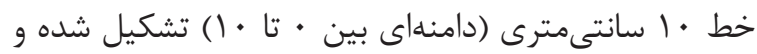

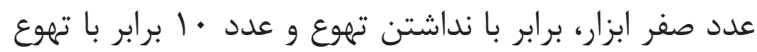

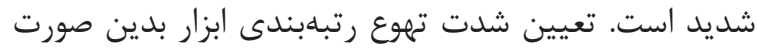

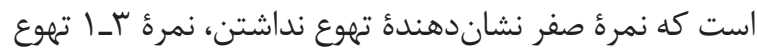

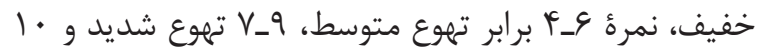

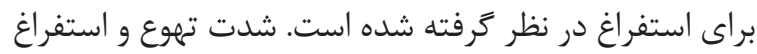

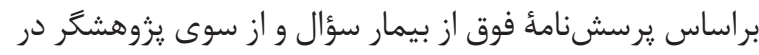

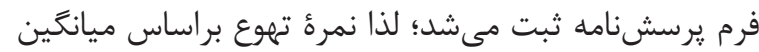

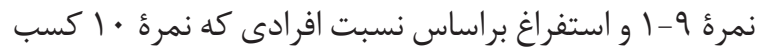

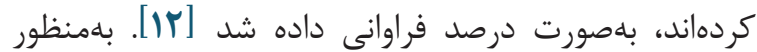

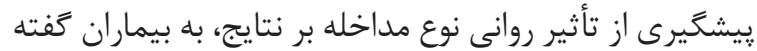

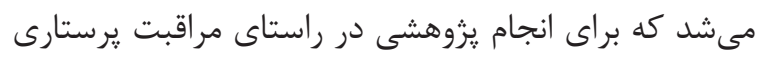

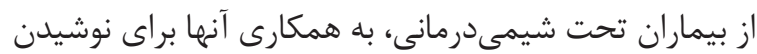

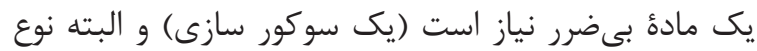

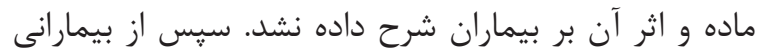

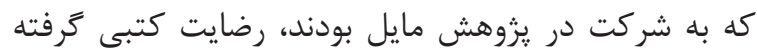

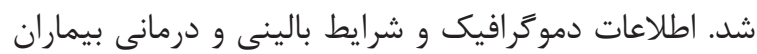

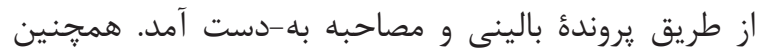

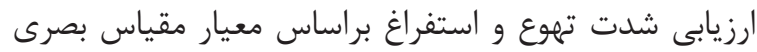

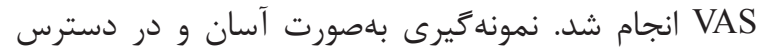

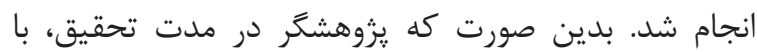

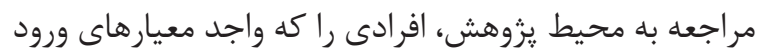

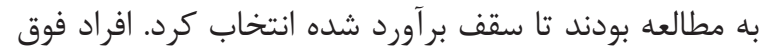

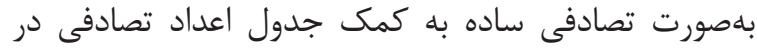

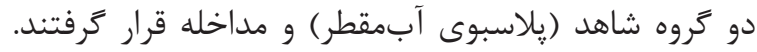

درمان مىشود [F]. تمهوع و استفراغ غيرقابل كنترل مى تواند

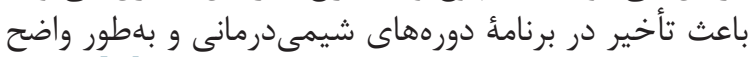

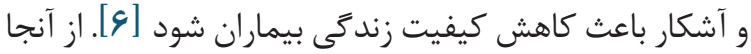

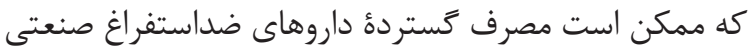

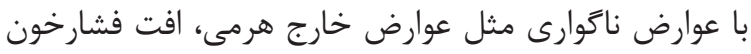

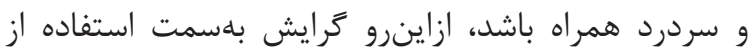

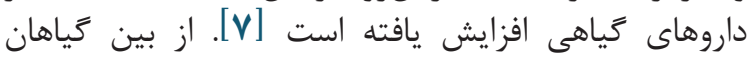

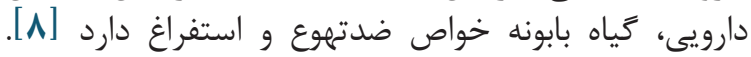

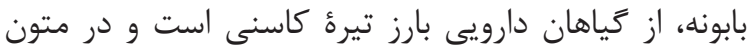

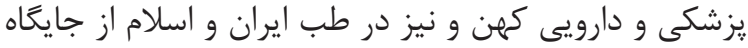

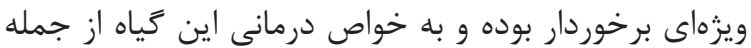

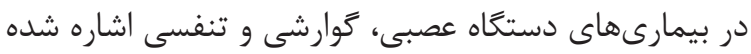

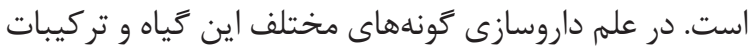

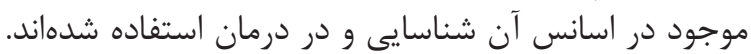

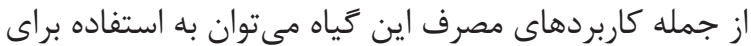

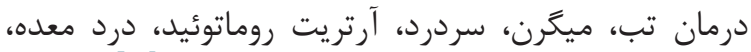

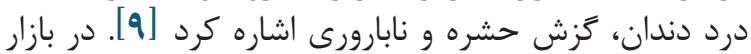

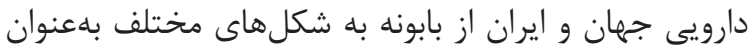

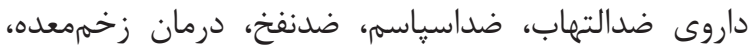

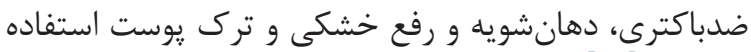

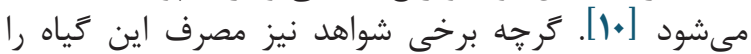

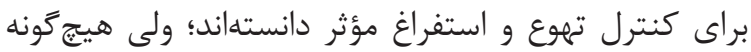

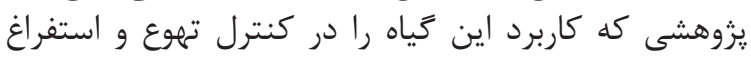

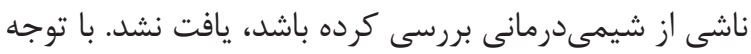

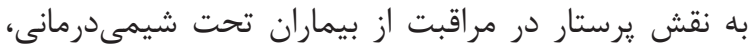

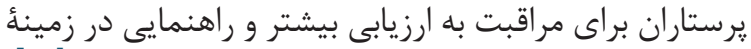

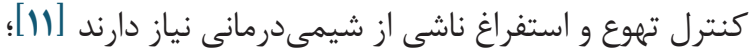

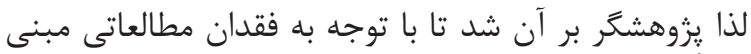

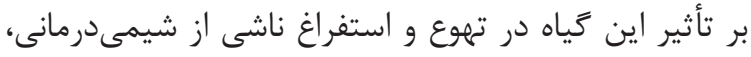

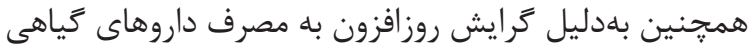

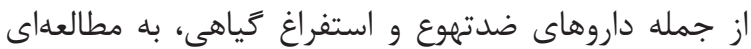

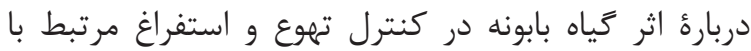

شيمى درمانى بيردازد.

\section{روش كار}

اين مطالعهُ كارآزمايى بالينى در سال جوجا در بيمارستان

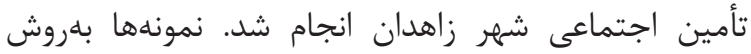

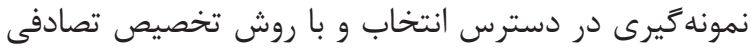

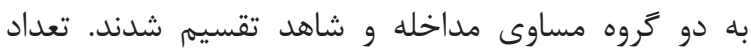

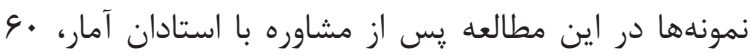
نفر بيمار مبتلا به سرطان تحت شيمى درمانى محاسبه شد.

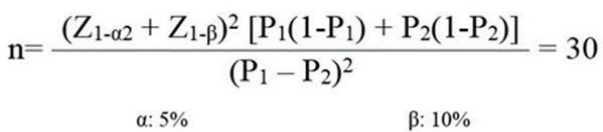

$$
\begin{aligned}
& \text { n : تعداد نمونهٔ مدنظر: • } \\
& \text { Z: توضع نرمال استاندارد: 199 } \\
& \text { a: خطاى يذيرفتهشده : هـ • • }
\end{aligned}
$$


داد كه تفاوت معنى دارى بين دو گروه مداخله و شاهد وجود نداشته است.

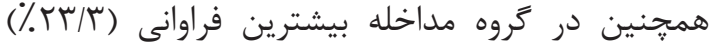

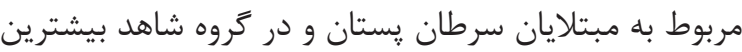

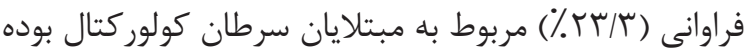

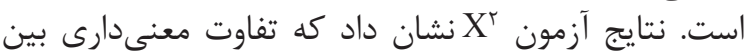

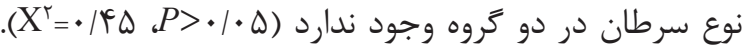
(جدول شماره r)

براساس جدول شماره با، قبل از شيمى شرمانى بيشترين ميانگين نمره تهوع (سT/

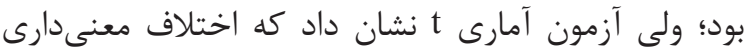

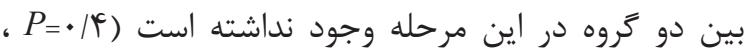

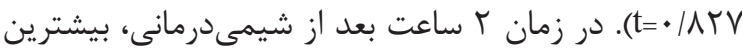

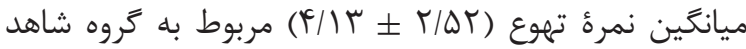

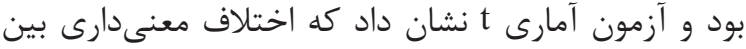

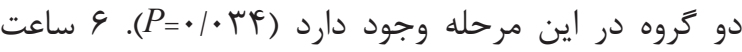

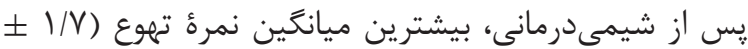

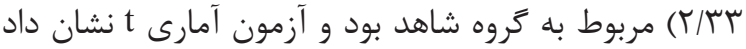
كه اختلاف معنى

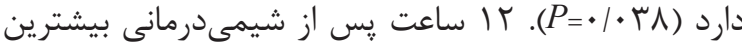

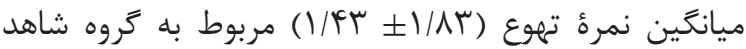

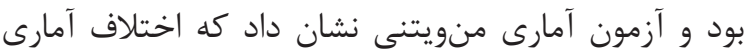

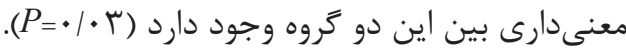

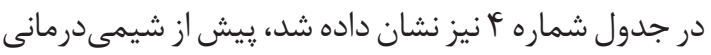

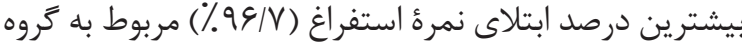

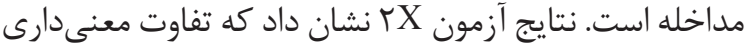

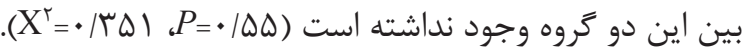

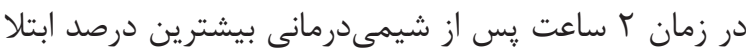

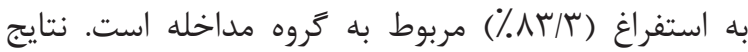

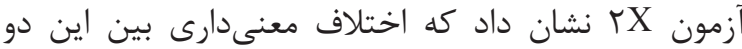

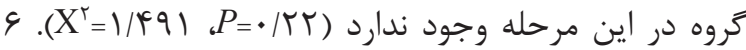

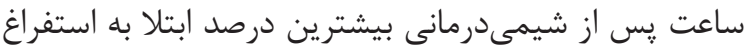

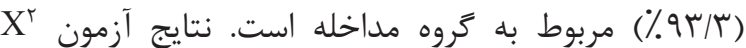

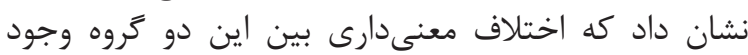

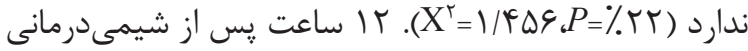

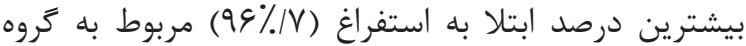

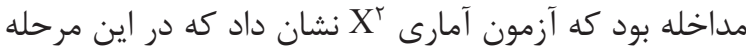

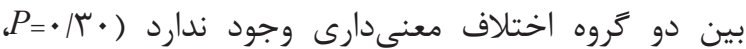

. $\left(X^{r}=\right) / \cdot V$
شيمى درمانى همةٔ شر كت كنند

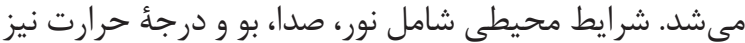

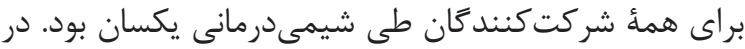

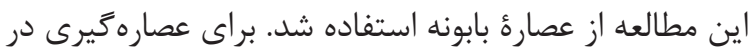

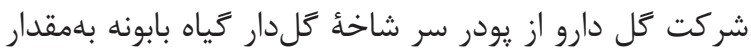

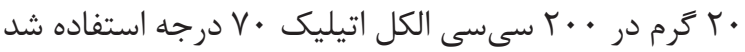

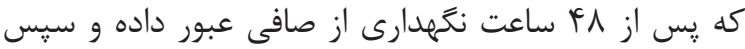

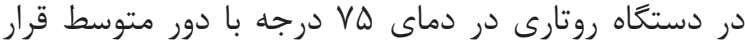

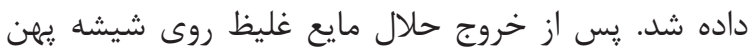

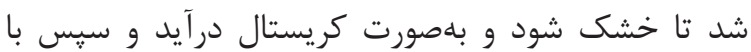

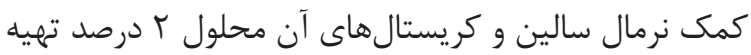

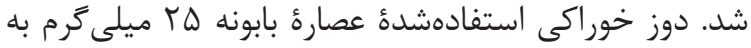

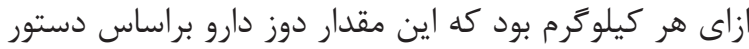

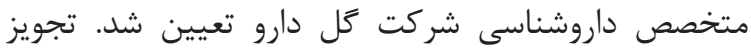

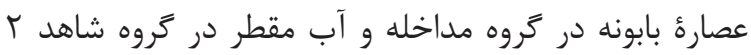

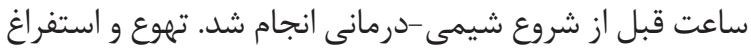

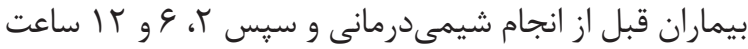

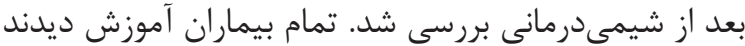

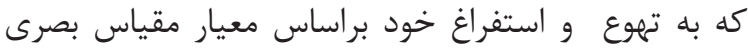

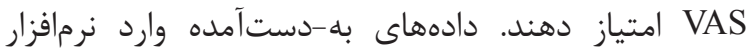

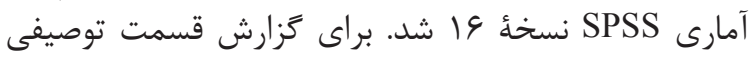

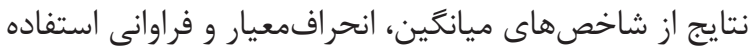

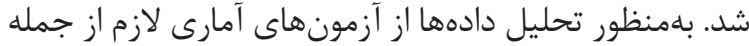

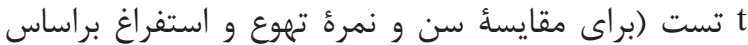

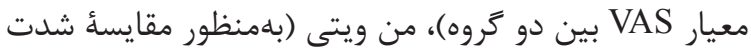

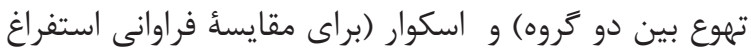

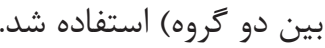

\section{يافتهها}

نتايج اين مطالعه نشان داد در زروه مان مداخله بيشترين

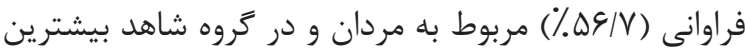

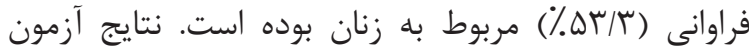

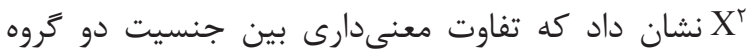

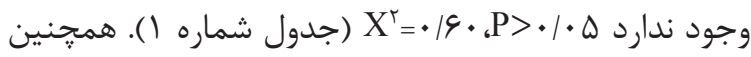

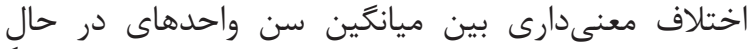

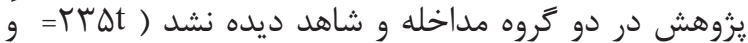

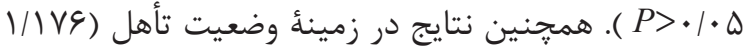

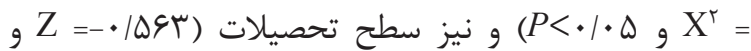

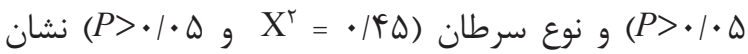

جدول شماره ا. توزيع فراوانى (تعداد و درصد) بيماران شركتكننده در مطالعه برحسب گروهِ در حال مطالعه و جنسيت

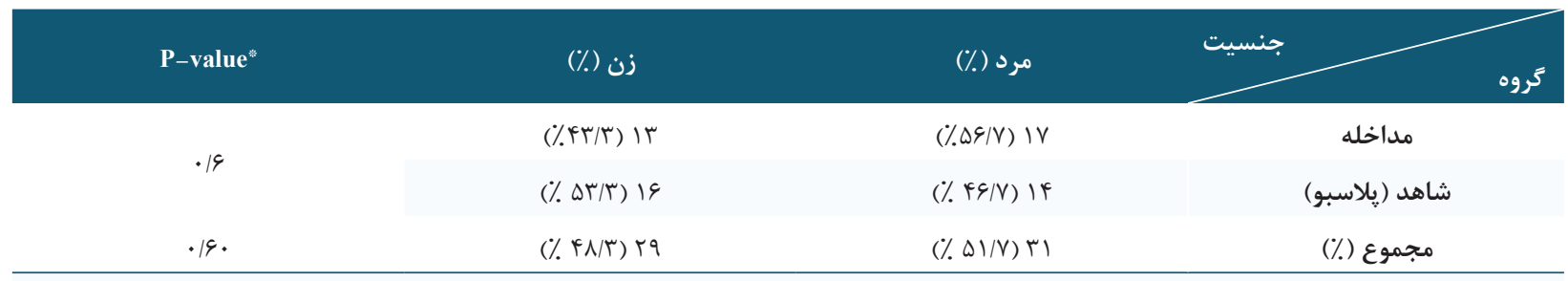

"Chi-square test 
جدول شماره r. توزيع فراوانى (تعداد ـدرصد) بيماران شركت كننده در مطالعه برحسب تروه درحال مطالعه و نوع سرطان

\begin{tabular}{|c|c|c|c|c|}
\hline P-value & مجموع (\%) & شاهد (يلاسبو) (\%) & مداخله (\%) & كرو \\
\hline \multirow{7}{*}{.199} & $(\% / V) \mid r$ & $(\% r \cdot)^{4}$ & $(\% r r / r) \vee$ & سرطان يستان \\
\hline & $(\% r \mid / V) \mid r$ & $(\% T / r) \vee$ & $(\% \cdot \cdot)^{4}$ & سرطان كولور كتال \\
\hline & $(\% \mid r / \mu) \wedge$ & $(\% \mid \varepsilon / V) \Delta$ & $(\% \cdot)^{\mu}$ & سرطان ريه \\
\hline & $(\% \mid 9 / V) 1$. & $(\% 19 / V) \Delta$ & $(\% 19 / V) \Delta$ & لوكمى \\
\hline & $(\% / / \Gamma) \Delta$ & $(\% / \mathrm{V}) \mathrm{r}$ & $(\%) \mu$ & سرطان بيضه \\
\hline & $(\% \wedge / \Gamma) \backslash 1$ & $(\% \mid g / V) \Delta$ & $(\% \cdot \cdot)^{4}$ & ساير \\
\hline & $(\%) \cdots)^{4}$. & $(\%) \cdots) \mu$. & $(\%), \cdots$ r. & مجموع (\%) \\
\hline
\end{tabular}

جدول شماره r. ميانگين و انحرافمعيار نمره تهوع (VAS) ناشى از شيمىدرمانى برحسب گروه در حالِ مطالعه و زمان ارزيابى

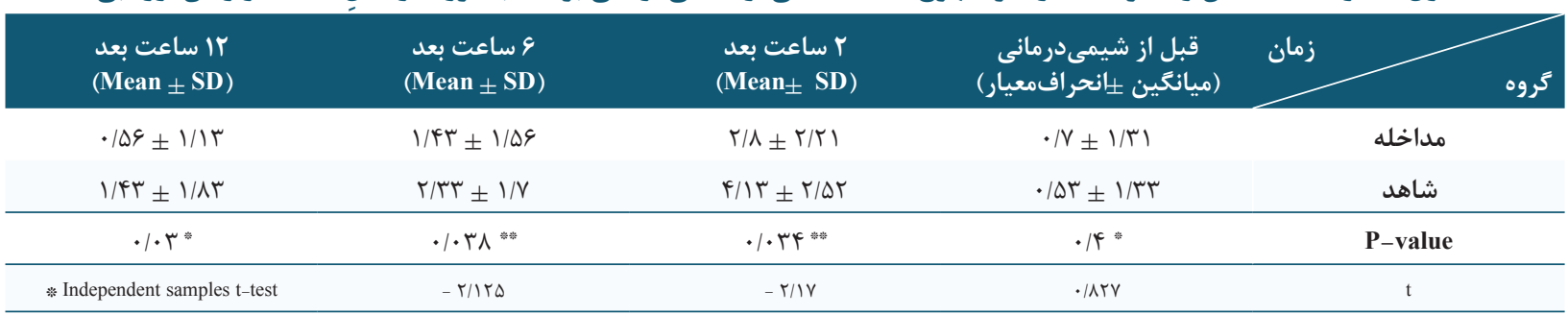

جدول شماره f. فراوانى (تعداد ـ درصد ابتلا) استفراغ ناشى از شيمىدرمانى بين كروههاى مطالعه برحسب زمان ارزيابى

\begin{tabular}{|c|c|c|c|c|c|}
\hline 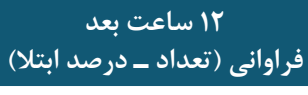 & فراوانى (تعداد ــدرصد ابتلا) & 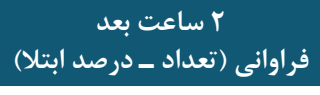 & فراوانى (تعداد ـ درصد از شرمانى ابتلا) & زمان & كروه \\
\hline$(\% ৭ 9 / V)$ ४ & $(\% q Y / T) Y \Lambda$ & $(\% \wedge r / r) r \Delta$ & $(\% ৭ 9 / V) \curlyvee q$ & دارد & \multirow{3}{*}{ مداخله } \\
\hline (\%/r) & $(\% g / V) r$ & $(\% 19 / V) \Delta$ & $(\% / r) 1$ & ندارد & \\
\hline$(\%) \cdot)^{\mu}$ & $(\%) \cdot{ }^{\prime}$ & $(\%) \cdots$ & $(\%) \cdots{ }^{\prime}$ & مجموع & \\
\hline$(\% q \cdot) T V$ & $(\% \wedge r / \Gamma) \Gamma \Delta$ & $(\% \cdot \cdot) r I$ & 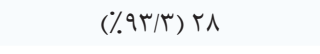 & دارد & \multirow{3}{*}{ شاهد } \\
\hline$(\% \cdot) r$ & $(\% 19 / V) \Delta$ & $(\% \cdot)^{9}$ & $(\% / \mathrm{V}) \mathrm{r}$ & ندارد & \\
\hline$(\%) \mu$. & $(\%) \cdot{ }^{\prime} \cdot$ & $(\% \cdots) r$. & $(\%) \cdot \cdots)$ r. & مجموع & \\
\hline$\cdot / \mu$ & $\cdot / T T$ & . RT & $\cdot 1 \Delta \Delta$ & \multicolumn{2}{|c|}{ P-value } \\
\hline
\end{tabular}

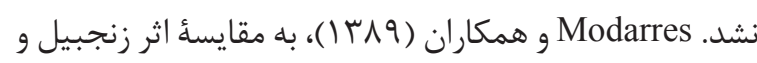

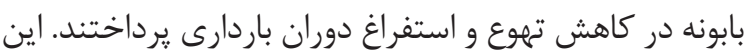

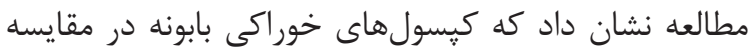

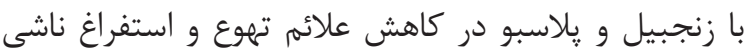

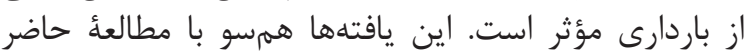

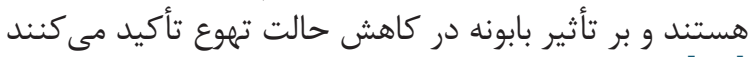

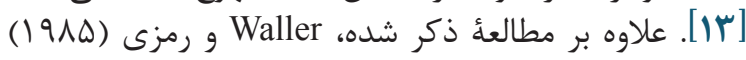

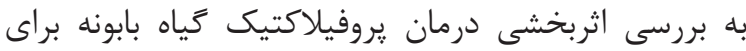

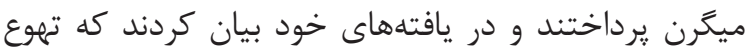

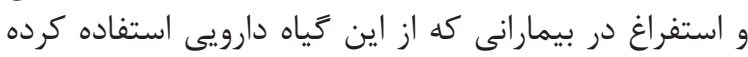

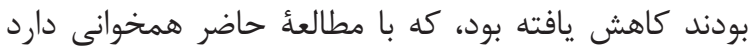

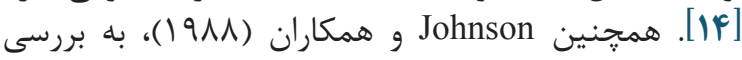

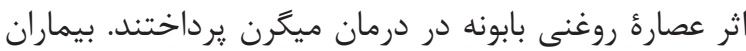

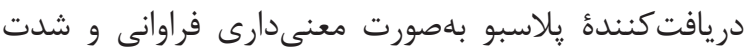

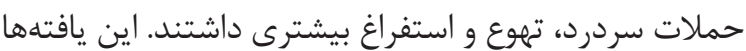

\section{بحث}

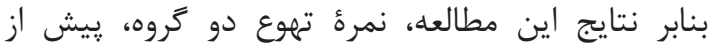

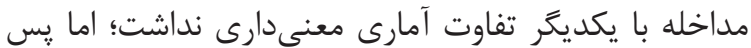

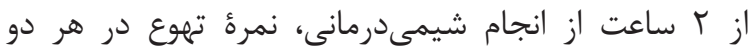

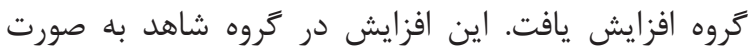

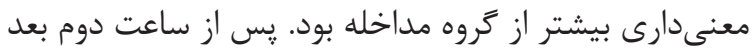

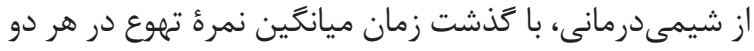

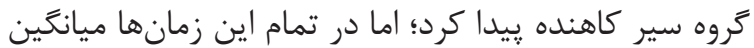

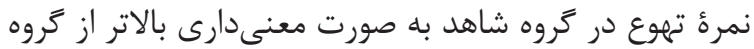

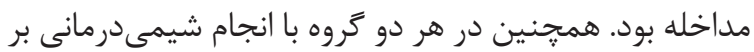

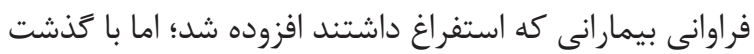

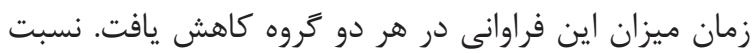

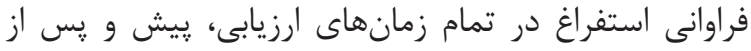

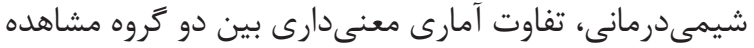


انحراف معيار تهوع دو كروه وجود دارد. همرجنين نتايج آزمون

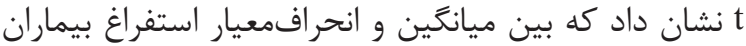

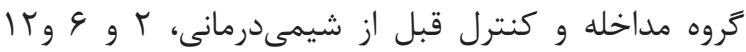

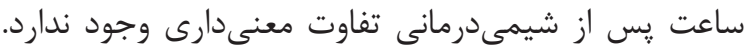

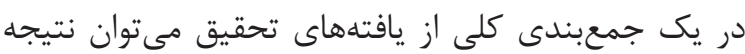

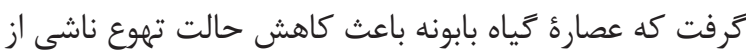

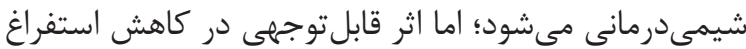

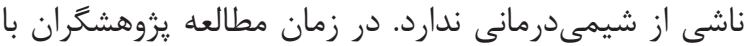

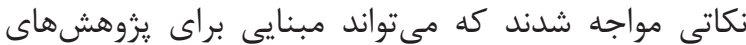

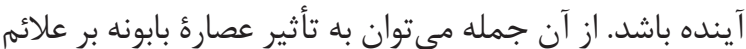

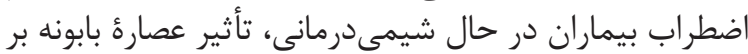

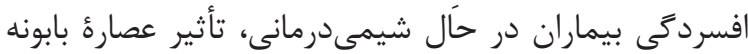

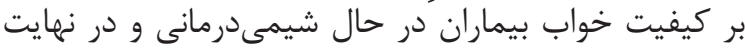

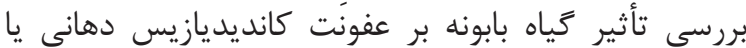

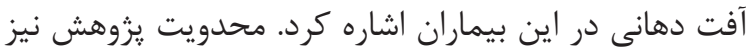

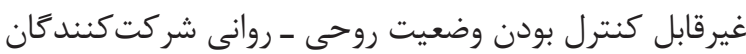

\section{سياسگزارى}

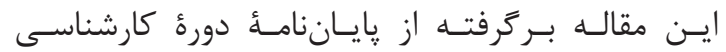

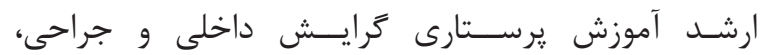

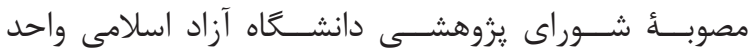

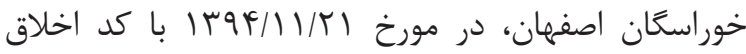

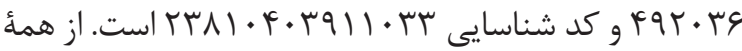

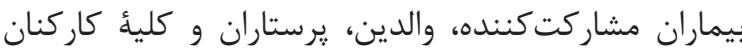

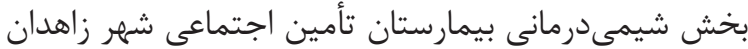
تشكر و قدردانى ويزه بهعمل مى بيماريد تأمين اجنان

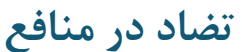

بين نويسندكان هيكونه تعارضى در منافع تزارش نشده
مطرحكننده اين است كه اين درمان علاوه بر كاهش فراوانى

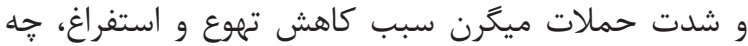

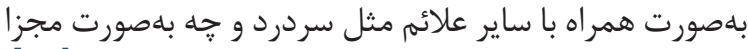

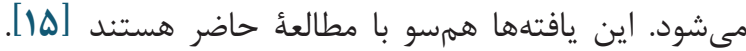

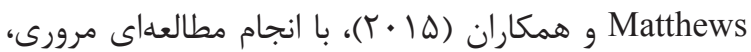

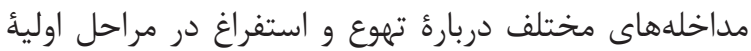

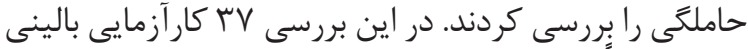

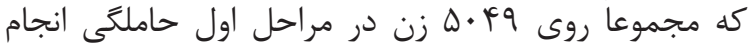

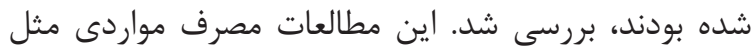

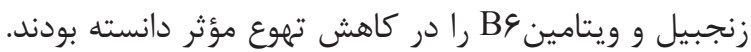

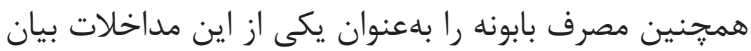

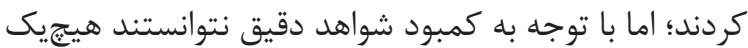

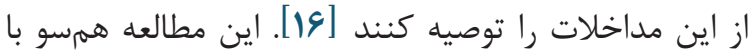

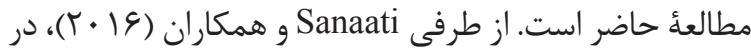

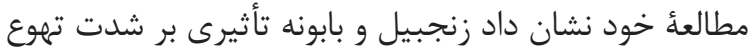

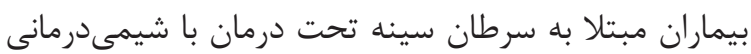

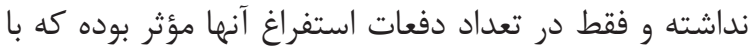

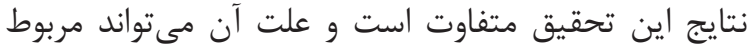

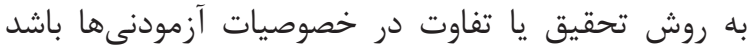

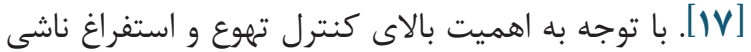

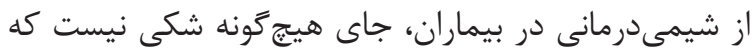

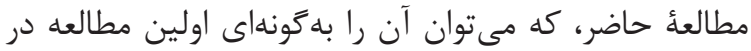

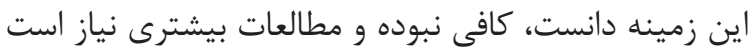

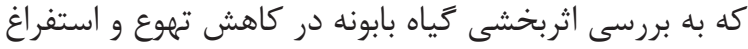
ناشى از شيمىدرمانى يرداخته شود.

$$
\text { نتيجه تيرى }
$$

در رابطه با فرضية يزووهش، يعنى بين ميزان تهوع و استفراغ

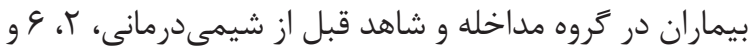

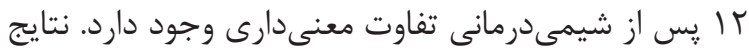

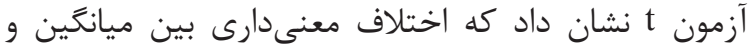

\section{References}

1. Naseri N, Taleghani F. Social support in cancer patients referring to Sayed Al-Shohada Hospital. Iranian journal of nursing and midwifery research. 2012;17(4):279283. PMid:23833627 PMCid:PMC3702147

2. Braunwald E, Fauci AS, Kasper DL, Hauser SL, Longo DL, Jameson JL. Harrison's Principles of internal medicine.1th edission, Booston: MC Graw. Hill; 2012.

3. 3.Roffe L, SchmidtK, ErnstE.Asystematic review of guided imagery as an adjuvant cancer therapy. Psycho-Oncology J. 2005;14(8):607-17. https:// doi.org/10.1002/pon.889

PMid:15651053

4. Shinomiya $\mathrm{K}$, Inoue $\mathrm{T}$, Utsu $\mathrm{Y}$, Tokunaga $\mathrm{S}$, Masuoka T, Ohmori A and et al. Hypnotic activities of chamomile and passiflora extracts in sleep-disturbed rats. Biological and Pharmaceu- tical Bulletin. 2005;28(5):808-10. https://doi. org/10.1248/bpb. 28.808

PMid:15863883

5. Henry DH, Viswanathan HN, Elkin EP, Traina S, Wade S, Cella D. Symptoms and treatment burden associated with cancer treatment: results from a cross-sectional national survey in the US. Supportive care in cancer J. 2008;16(7):791-801. https://doi.org/10.1007/ s00520-007-0380-2 PMid:18204940

6. Navari RM. Management of chemotherapy-induced nausea and vomiting pediatric pationts . Pediatric Drugs J. 2017;19 (3):213-222. $\quad$ https://doi.org/10.1007/ s40272-017-0228-2

PMid:28447301

7. Hickok JT, Roscoe JA, Morrow GR, Ryan JL. A phase II/III randomized, placebo-controlled, double-blind clinical trial of ginger (Zingiber officina- 
le) for nausea caused by chemotherapy for cancer. Supportive cancer therapy J. 2007;4(4):247-50. https://doi.org/10.3816/ SCT.2007.n.022

PMid:18632524

8. Modares M, Besharat S, Mahmoudi M. Effect of Ginger and Chamomile capsules on nausea and vomiting in pregnancy. Journal of Gorgan University of Medical Sciences. 2012;14(1):46-51.

9. Pareek A, Suthar M, Rathore GS, Bansal V. Feverfew (Tanacetum parthenium L.): A systematic review. Pharmacognosy reviews. 2011;5(9):103. https://doi.org/10.4103/0973-7847.79105 PMid:22096324

PMCid:PMC3210009

10. Ebrahimi S, Pormohammadi A.comprehenssion effective bittween Mtricaria and black tea redused instabilityintants when grow their thooth. Dena Quarterly periodical, 2010;4(1):23-30.

11. Krishnasamy M, Annab R, Wisniewski T. The Nurse's Role in Managing Chemotherapy-Induced Nausea and Vomiting: An International Survey. Cancer nursing. 2014;37(4):27-35. h https://doi.org/10.1097/ NCC.0b013e3182a3534a PMid:24145248

12. Bodian CA, Freedman, G, Hossain S, Eisenkraft J, Beilin Y. The visual analog scale for pain:

$$
\text { 4 أ. بررسى تأثير عصارة گياه بابونه بر شدت تهوع و... }
$$

clinical significancein postoperative patients. Anesthesiology J. 2001;95(6):1356-61. https:// doi.org/10.1097/00000542-200112000-00013

13. Modarres M, Besharat S,Mahmodi M. Omprehenssion effective bittween Mtricaria oral capsul of Ginger and Mtricaria on Nausea and Vomiting of pregnancy period. Gorgan medical science university J. 2012;14(1)-:46-51.

14. Waller P, Ramsay L. Efficacy of feverfew as prophylactic treatment of migraine. $\mathrm{Br}$ Med J (Clin Res Ed). 1985;291(6502):1128-32. https://doi.org/10.1136/bmj.291.6502.1128

15. Johnson ES, Hylands PJ, Oil extract of tanacetum parthenium for treating migraine. http:// www.patents.com/us-4758433.html[ Online]

16. Matthews A, Haas DM, Dowswell T. Interventions for nausea and vomiting in early pregnancy. The Cochrane Library. 2015. https:// doi.org/10.1002/14651858.CD007575.pub4

17. Sanaati F, Najafi S, Kashaninia Z, Sadeghi M. Effect of Ginger and Chamomile on Nausea and Vomiting Caused by Chemotherapy in Iranian Women with Breast Cancer. Asian Pac J Cancer Prev, 2016;17(8):4125-9. PMid:27644672 